\title{
Plasmonic Resonance toward Terahertz Perfect Absorbers
}

\author{
Withawat Withayachumnankul, ${ }^{\dagger}{ }^{\dagger}$ Charan Manish Shah, ${ }^{\ddagger}$ Christophe Fumeaux, ${ }^{\dagger}$ Benjamin S.-Y. Ung, ${ }^{\dagger, \perp}$ \\ Willie J. Padilla, ${ }^{\$}$ Madhu Bhaskaran, ${ }^{\ddagger}$ Derek Abbott, ${ }^{\dagger}$ and Sharath Sriram*, $*$ \\ ${ }^{\dagger}$ School of Electrical and Electronic Engineering, The University of Adelaide, Adelaide, SA 5005, Australia \\ ${ }^{\ddagger}$ Functional Materials and Microsystems Research Group, School of Electrical and Computer Engineering, RMIT University, \\ Melbourne, VIC 3001, Australia \\ ${ }^{\S}$ Department of Physics, Boston College, 140 Commonwealth Avenue, Chestnut Hill, Massachusetts 02467, United States
}

ABSTRACT: Metamaterial perfect absorbers have garnered significant interest with applications in sensing, imaging, and energy harnessing. Of particular interest are terahertz absorbers to overcome the weak terahertz response of natural materials. Here, we propose lossy plasmonic resonance in silicon-based annular microcavities for perfect terahertz absorption. This mechanism is in stark contrast to earlier demonstrations of conventional terahertz perfect absorbers that invoke Lorentzian electric and magnetic resonances. A fundamental cavity mode coupled to coaxial surface plasmon

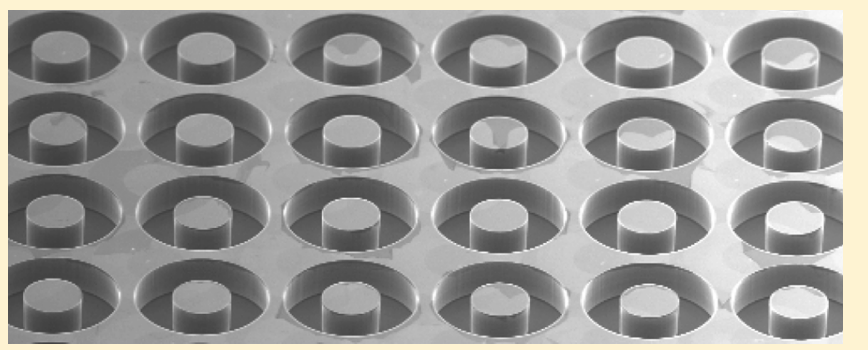
polaritons is responsible for the predicted exceptional absorption of $-58 \mathrm{~dB}$ with a $90 \%$ absorption bandwidth of $30 \%$. The performance is in agreement with experimental validation and consistent with critical coupling and resonance conditions. This specific cavity design possesses great thermal isolation and minimal electromagnetic coupling between unit cells. These unique features exclusive to the plasmonic cavity introduce a promising avenue for terahertz imaging with enhanced contrast, resolution, and sensitivity.

KEYWORDS: metamaterial, plasmonics, perfect absorber, cavity mode, THz-TDS, terahertz, multiphysics simulation

$\mathrm{R}$ esearch on perfect absorption has recently become a very active topic in the rapidly growing field of metamaterials. Providing enhanced performance and flexibility, perfect absorbers have been realized in different applications, including imaging, sensing, and energy harnessing. Soon after the seminal development by Padilla and co-workers, ${ }^{1,2}$ metamaterial-based perfect absorbers have been demonstrated across the spectrum, from the microwave, to terahertz, infrared, and optical bands. ${ }^{3}$ In general, these traditional perfect absorbers appeared in the form of metallic resonators on a ground plane, designed to eliminate the reflection and enhance the absorption. The perfect absorption mechanism was explained with the coexistence of Lorentzian electric and magnetic responses that match the impedance of the structure to free space and at the same time imposing large energy dissipation on resonance. Another explanation involved wave interference theory where multipath reflections originating from different interfaces lead to completely destructive interference in the direction of reflection. ${ }^{4}$ Alternatively, perfect absorption was interpreted as nullified reflection by an out-of-phase wave reradiated from induced electric and magnetic surface currents. ${ }^{5}$

Of relevance to this article are perfect absorbers at infrared and visible frequencies, where metals with relatively limited conductivity can support bound surface waves or surface plasmon polaritons (SPPs). Earlier implementations of plasmonic absorbers in the near-infrared ${ }^{6,7}$ and visible 8,9 regimes adopted the conventional design of a metallic dipole array on a ground plane separated by a dielectric spacer. This concept was extended to random nanoparticles on a ground plane that exhibit electric and magnetic resonances for visible light absorption. ${ }^{5,10}$ Unlike perfect absorbers at lower frequencies, where loss occurs mainly inside the dielectric spacer, energy dissipation in these plasmonic absorbers takes place in the lossy metallic components. Other realizations were based on a solid metal sheet with periodic textures, including nanovoids $^{11,12}$ and nanogrooves. ${ }^{13-15}$ Depending on the geometries and dimensions, these textured metallic surfaces can exhibit perfect absorption via either localized surface plasmon resonances inside cavities or delocalized in-plane SPP coupling.

At terahertz frequencies, various types of plasmonic resonators, including dipoles, bowties, and coupled disks, constructed from doped semiconductors have been demonstrated for localized surface plasmon resonances. ${ }^{16-19}$ Nonetheless their absorption performance has never been explored in this frequency range. In this article, a terahertz perfect absorber based on plasmonic resonance is proposed. The design is inspired by our earlier demonstration of complementary plasmonic resonators in the form of annular cavities etched into the surface of a doped silicon wafer. ${ }^{20}$ These resonant cavities exhibit relatively strong nonradiative damping,

Received: April 7, 2014

Published: June 3, 2014 
essential for efficient energy trapping. With dimensions satisfying the critical coupling condition, the cavity array can absorb nearly $100 \%$ of incident terahertz energy at resonance. This article discusses the mechanism of plasmonic resonances in microcavities, approaches for fabrication and measurement for experimental validation, and observable electromagnetic and thermal responses. Importantly, the conditions of resonance and critical coupling are analyzed theoretically in relation to a coaxial plasmonic mode.

\section{RESONANCE MECHANISM}

Figure 1 depicts a single annular cavity, etched straight down into the surface of a semiconductor wafer. The unit cell size is on the order of the operating wavelength, the cavity depth is about a quarter wavelength, and the annular gap is on the subwavelength scale. The supporting semiconductor is moderately doped with its plasma frequency in the terahertz regime. Linearly polarized terahertz waves incident normally to the surface are diffracted by the subwavelength annular gap. This diffraction results in phase matching to SPPs that can be sustained along the cavity sidewalls. Since the sidewall separation is less than the decay length of the normal electric field component, the confined SPPs form a coupled mode that is controlled by the gap size. Effectively, the excited SPPs establish the first-order coaxial SPP mode, as indicated by the red arrow in Figure 1a. This mode is a plasmonic counterpart of the $\mathrm{TE}_{11}$ mode in perfect-electric-conductor (PEC) coaxial waveguides. The excited SPPs propagate back and forth along the cavity axis. A resonance resulting from a standing wave can be observed in the reflection spectrum and can be tuned via the cavity depth and the gap size. Owing to the truly localized mode, electromagnetic coupling between adjacent cavities is minimal. It is worth noting that the resonance mechanism is related to different gap plasmon modes observed in textured metal surfaces at the infrared and visible regimes. ${ }^{12,13}$

\section{RESULTS AND DISCUSSION}

This section discusses numerical and experimental results and elucidates the cavity absorption performance in terms of electromagnetic functionality and corresponding thermal behavior.

Electromagnetic Behavior. Figure 2a shows the reflection amplitude response of the fabricated cavity array at a normal angle of incidence. The measurement and simulation are in

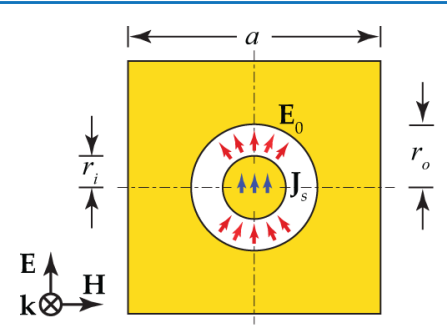

(a)

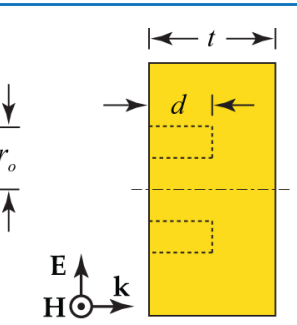

(b)
Figure 1. Schematic of the plasmonic cavity resonator for perfect absorption. A unit cell is illustrated from (a) the top view and (b) the side view. The colored areas represent doped semiconductor. The red arrows indicate induced instantaneous electric field $\mathbf{E}_{0}$, and the blue arrows represent the electric current density $\mathbf{J}_{s}=\hat{n} \times \mathbf{H}$. The designed dimensions are as follows: $a=200 \mu \mathrm{m}, d=60 \mu \mathrm{m}, r_{i}=40 \mu \mathrm{m}, r_{0}=$ $90 \mu \mathrm{m}$, and $t=270 \mu \mathrm{m}$. The schematic is not to scale.
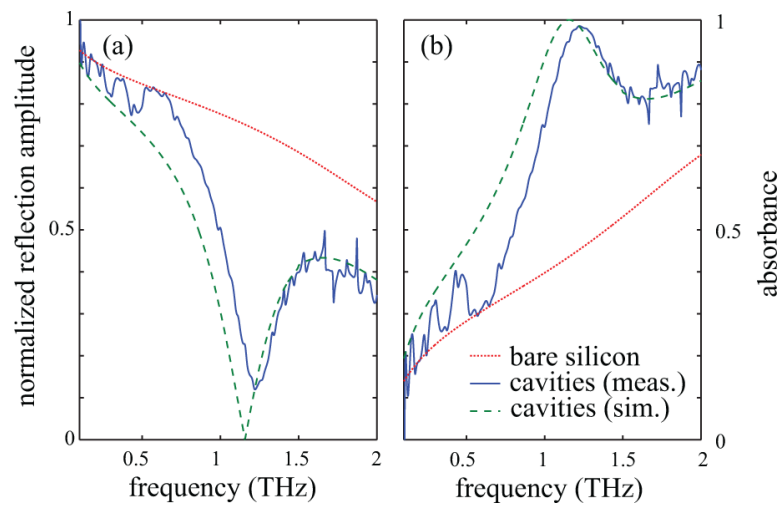

Figure 2. Reflection and absorbance spectra. (a) Normalized reflection amplitude spectra, $S_{11}$, and (b) power absorbance spectra, $A=1-$ $\left|S_{11}\right|^{2}$. The numerical baseline spectra for a bare doped-silicon wafer are included for comparison. The numerical results are obtained from CST Microwave Studio 2013 with the frequency-domain solver. In simulation, the unit cell boundary condition is applied to all transverse boundaries, and the Drude model with measured silicon properties is implemented for the doped silicon. ${ }^{20}$

general agreement with small discrepancies associated with fabrication tolerances, optical alignment, and nonplanar wavefront of the incident wave. A strong resonance can be observed at around $1.16 \mathrm{THz}$ with a numerically resolved minimal reflection of $-58 \mathrm{~dB}$, compared with a measured reflection of $-18 \mathrm{~dB}$ at $1.22 \mathrm{THz}$. The bare silicon wafer shows a baseline reflection profile with monotonically decreasing amplitude toward high frequencies, where the silicon becomes dielectric with a constant reflection amplitude of around 0.55 . No terahertz transmission through the cavity sample is detectable within the available system bandwidth and dynamic range. Additional simulation suggests that the sample thickness, $t$, for the cavity array can be as thin as $80 \mu \mathrm{m}$ with a transmission level below $-25 \mathrm{~dB}$. The simulated and measured absorbance profiles are shown in Figure $2 \mathrm{~b}$. At resonance, the simulated and measured absorbance levels are about $100 \%$ and $98.5 \%$, respectively. From the simulation, the $90 \%$ absorption bandwidth is around $360 \mathrm{GHz}$, equivalent to $30 \%$, compared with $290 \mathrm{GHz}$ or $24 \%$ observed from the measurement. The strong absorption is caused by field interaction and ohmic loss in the doped silicon, in contrast to the dominant dielectric loss in conventional perfect absorbers at terahertz frequencies and below. ${ }^{3}$

The absorbance maps in response to oblique incident waves are shown in Figure 3. It is clear that the absorption performance can be maintained up to an angle of about $35^{\circ}$ for both polarizations. Beyond this angle, the absorption degrades rapidly for the TE polarization, as the $\mathrm{TE}_{11}$ coaxial mode is weakened. For the TM polarization, the mode and hence the absorption efficiency are reasonably sustained. Other modes are excited in both polarizations at large angles of incidence. The observed absorption performance coincides with that of most conventional perfect absorbers, where the $\mathrm{TM}$ excitation preserves a wide range of absorption up to $80^{\circ}$, and the absorptivity for the TE excitation diminishes beyond $40^{\circ}{ }^{3}$ In the case of annular cavities, the effect can be described by the balance of the electric field distribution on the left and right sides of the $\mathbf{E}$ plane. The imbalanced electric field distribution for the TE-polarized excitation results in weak coupling to the $\mathrm{TE}_{11}$ mode at oblique incidence angles. The effect can also be explained in terms of equivalent sources on 
(a) TE polarization

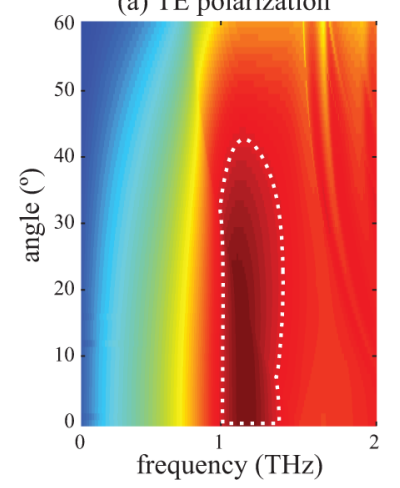

(b) TM polarization

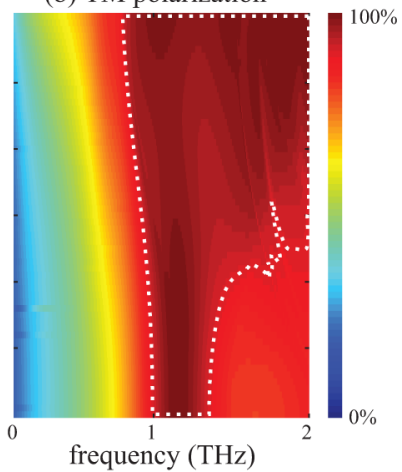

Figure 3. Absorbance maps at oblique angles of incidence. The maps are a function of frequency and angle for (a) TE polarization and (b) TM polarization. The dotted contours define the areas where the absorbance is above $90 \%$. The results are obtained from the CST Microwave Studio frequency-domain solver with Floquet ports and the unit-cell boundary condition.

the surface of the central pillar. For the TE polarization, the incident magnetic field component tangential to the surface is not symmetric with respect to the $\mathbf{E}$ plane and, hence, leads to an imbalanced distribution of the surface current density $\mathbf{J}_{s}$.

Resonance and Coupling Conditions. In the case of normal incidence, the resonance and coupling conditions can be determined from the dispersion of the first-order coaxial SPP in an equivalent plasmonic coaxial waveguide, as shown in Figure 4. Due to strong field confinement, the dispersion curve of the plasmonic waveguide, $\beta^{\prime}$, lies to the right of the PEC waveguide dispersion curve. Deduced from $\beta^{\prime \prime}$, a decrease in the propagation length toward higher frequencies is ascribed to increased field confinement, while at low frequencies a reduction in the propagation length is due to increased field penetration into the lossy silicon. For the coaxial cavity, the fundamental cavity mode needs to satisfy the standing-wave condition $2 d \beta^{\prime}=0.9 \pi$, or $\beta^{\prime}=23562 \mathrm{rad} / \mathrm{m} .^{20}$ It is noted that the $0.9 \pi \mathrm{rad}$ accounts for a reflection phase change at the

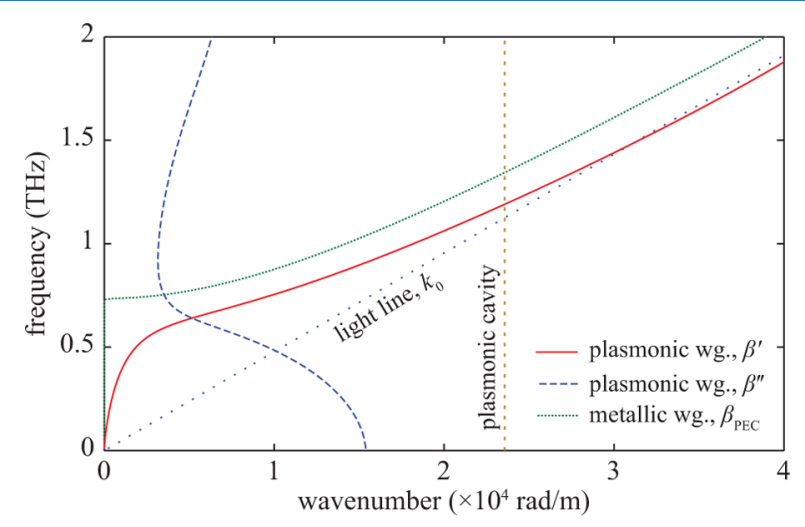

Figure 4. Dispersion relation of equivalent plasmonic coaxial waveguide. The waveguide has the same inner and outer radius as the cavity shown in Figure 1. The complex wavenumber $\beta=\beta-j \beta^{\prime \prime}$ is associated with the first-order coaxial SPP mode in the plasmonic waveguide. ${ }^{21}$ The dispersion for a PEC coaxial waveguide counterpart operating in the $\mathrm{TE}_{11}$ mode is included for comparison. The vertical line marks the resonance condition for the plasmonic cavity with a depth of $60 \mu \mathrm{m}$. The imaginary part of the wavenumber is related to the propagation length via $L=\left(2 \beta^{\prime \prime}\right)^{-1}$. The effective complex permittivity is defined as $\hat{\varepsilon}_{\text {eff }}=\left(\beta / k_{0}\right)^{2}$. bottom of the cavity, observed numerically. From Figure 4, this wavenumber corresponds to the frequency of $1.2 \mathrm{THz}$, in good agreement with the resonance frequency observed from the experimental and numerical results. It is noted that although the resonance requires a quarter-wavelength cavity depth similar to conventional non-metamaterial absorbers, ${ }^{3}$ the physical depth constraint can be relaxed through plasmonic confinement and, if necessary, though a dielectric medium loading into the air gap.

On resonance, if all the incident energy is dissipated inside the resonator, the reflected energy vanishes: the so-called critical coupling effect. For opaque structures, the critical coupling condition can be established as $Q_{\text {eak }}^{-1}=Q_{\text {diss }}^{-1}$, where the $Q$ factors $Q_{\text {leak }}$ and $Q_{\text {diss }}$ are related with the leakage (radiation) and dissipation losses, respectively. ${ }^{22}$ The leakage $Q$ factor can be defined as $Q_{\text {leak }}^{-1}=v_{\mathrm{g}} T_{1} /\left(2 d \omega_{0}\right)$, where $T_{1}$ is the aperture transmittance, $\omega_{0}$ is the resonance angular frequency, and $v_{\mathrm{g}}=$ $\partial \omega_{0} / \partial \beta^{\prime}$ is the group velocity of waves inside the resonator. By assuming $T_{1}=1$ or the cavity aperture permits $100 \%$ transmission, $Q_{\text {leak }}^{-1}=0.26$. On the other hand, the inverse dissipation $Q$ factor equals $Q_{\text {diss }}^{-1}=\varepsilon_{\text {eff }}^{\prime \prime} /\left|\varepsilon_{\text {eff }}^{\prime}\right|=0.3$. Although the critical coupling condition is not exactly met, $Q_{\text {leak }}^{-1}$ and $Q_{\text {diss }}^{-1}$ are reasonably close and hence indicate efficient absorption of the plasmonic resonant cavities. The relatively large leakage and dissipation losses are responsible for the low- $Q$ resonance that results in a relatively wide absorption bandwidth of $30 \%$.

Thermal Behavior. Insight into the thermal performance of the plasmonic cavities can be inferred from electromagneticthermal cosimulation conducted using CST Microwave Studio 2013. Initially, electromagnetic simulation is used to resolve spatially distributed loss in the cavity at the resonance frequency. This can be computed with the time-domain solver with perfect electric and magnetic boundary conditions as transverse boundaries to simulate an infinite array. A planewave excitation impinges normally to the surface with a power of $10 \mathrm{~mW}$ per unit cell. The 3D steady-state loss distribution obtained from the electromagnetic simulation is then fed into the thermal cosimulation module, where the thermal stationary solver is employed to observe the cavity in the equilibrium state. The thermal conductivity of the phosphorus-doped silicon is $149 \mathrm{~W} / \mathrm{K} / \mathrm{m}$, compared with $0.026 \mathrm{~W} / \mathrm{K} / \mathrm{m}$ for air. ${ }^{23}$ Since the cavity array is uniform, symmetric, and excited at normal incidence, there is no heat flow across unit cells and between the two halves of a unit cell. Hence, an adiabatic boundary condition is applied to all transverse boundaries and onto the two symmetry planes. An isothermal boundary condition with a fixed temperature of $300 \mathrm{~K}$ is applied to the back silicon surface to imitate a heat sink. An open boundary condition placed at $300 \mu \mathrm{m}$ away from the cavity surface leaves the cavity exposed to room temperature set to $300 \mathrm{~K}$.

Figure 5 shows the steady-state thermal volume loss of the cavity at different frequencies. Clearly, the thermal loss is maximum on resonance at $1.16 \mathrm{THz}$ and becomes weaker off resonance. It can be inferred from Figure $5 b$ that on resonance most of the incident energy gets absorbed in the center pillar due to strong current induction associated with the coaxial SPP mode. Relatively weak thermal loss can be observed along the narrowed cavity edges, bearing high resistance for return current paths. The thermal loss on the bare silicon surface is merely $5.5 \times 10^{9} \mathrm{~W} / \mathrm{m}^{3}$, or over an order of magnitude lower than the maximum loss in the cavity, and hence not observable in Figure 5d. Inherent from strong thermal loss, the temperature increase is localized in the pillar, as shown in 

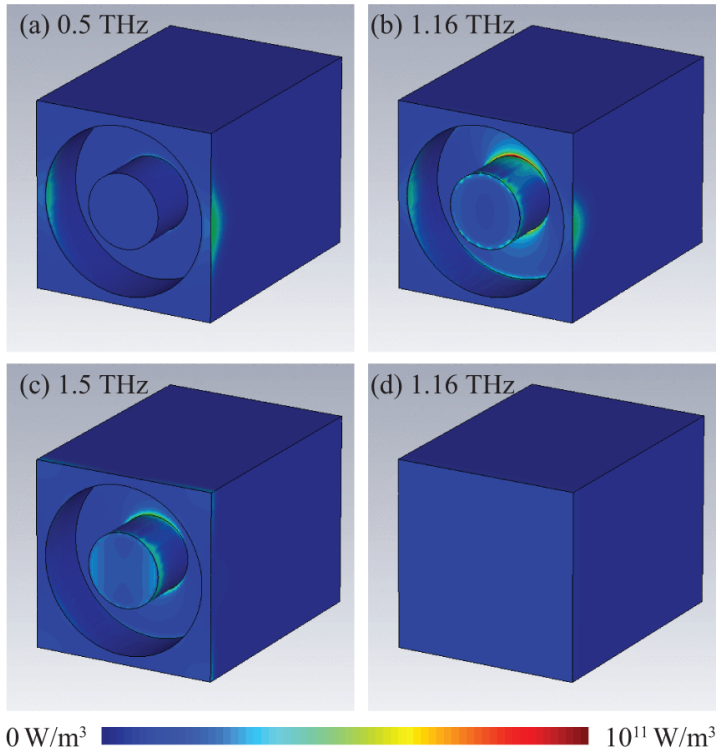

Figure 5. Thermal volume loss for plasmonic cavity and bare silicon substrate. $(\mathrm{a}-\mathrm{c})$ Plasmonic cavity at $0.5 \mathrm{THz}, 1.16 \mathrm{THz}$ (maximum absorption), and $1.5 \mathrm{THz}$, respectively. (d) Bare silicon substrate at $1.16 \mathrm{THz}$, with exactly the same settings and carrier concentration as the cavity. The color plots share the same linear scale.
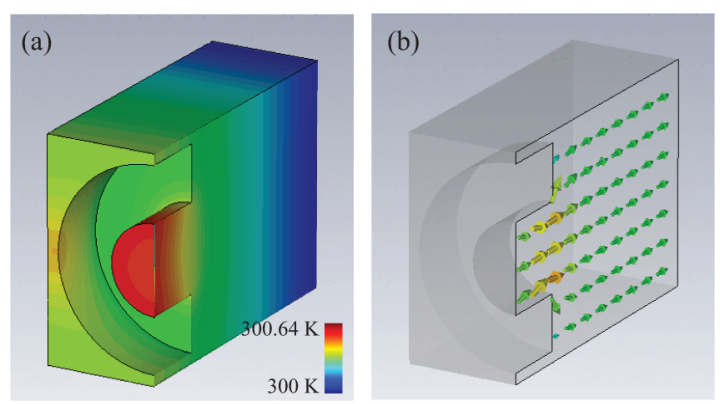

Figure 6. Steady-state temperature and heat-flow density of plasmonic cavity. Both the temperature (a) and heat-flow density (b) are calculated at the frequency of maximum absorption at $1.16 \mathrm{THz}$. The temperature color map is in a linear scale.

Figure 6a. The maximum temperature reaches $300.64 \mathrm{~K}$ and progressively decreases to $300 \mathrm{~K}$ toward the back of the substrate, where a heat sink is attached. It is worth noting that this level of temperature change does not significantly affect the carrier concentration ${ }^{23}$ and, hence, the resonance and absorption conditions. Since the pillar is surrounded by air with very low thermal conductivity, the heat can barely escape to the side and to adjacent unit cells, even in the case of a nonuniform excitation. Hence, heat accumulated in the pillar mainly flows toward the back in the silicon, as indicated by the heat-flow density in Figure $6 \mathrm{~b}$. The particularly high thermal conductivity of silicon benefits the thermal response time, defined as $\tau=C / \Lambda$, where $C$ is the heat capacity and $\Lambda$ is the thermal conductance. ${ }^{24}$ The response time can be reduced further by thinning the substrate to reduce the thermal mass and hence the heat capacity, yet maintaining the absorption performance.

\section{CONCLUSION}

In this article, nearly perfect absorption at terahertz frequencies has been demonstrated with plasmonic microcavities made of moderately doped silicon. The mechanisms underlying absorption are the first-order coaxial SPPs and fundamental standing waves developed inside the cavities. With the critical coupling satisfied, the incident wave energy is mostly converted into heat with theoretical absorption loss of $58 \mathrm{~dB}$. By leveraging large leakage and dissipation damping, a $90 \%$ absorption bandwidth of $30 \%$ can be achieved with a single resonance. On resonance, thermal loss takes place mainly inside the pillar, giving rise to a local temperature increase therein. The high thermal conductivity of silicon leads to a short thermal response time of the structure. Since the resonance frequency can be controlled via carrier concentration, the structure is ready for dynamic operation via carrier injection. ${ }^{25}$ The fabrication is relatively inexpensive with silicon as the only bulk raw material and no multilayer processing. The absorber is suitable for emerging terahertz communications to suppress undesirable multipath effects.

As a further application, this plasmonic cavity design can operate as an integrated detector for active uncooled microbolometers. It is worth mentioning existing microbolometers employing metamaterial absorbers in the terahertz $^{26}$ and mid-infrared ranges ${ }^{24,27}$; here, power-dependent temperature or resistance change in the absorbers can be read out via thermocouples, electrodes, or MEM devices. Likewise, for our plasmonic cavity, either of these devices can be integrated onto the pillar to sense a change in temperature or resistance. Importantly, this plasmonic absorber design possesses the unique features of large electromagnetic and thermal isolation between adjacent unit cells. These features, together with nearly $100 \%$ absorption, potentially lead to an array of pixels with high resolution, high contrast, and high sensitivity for terahertz imaging. Since the intercell coupling is minimal, the pixel density can be spatially adapted with no degradation in the local absorption performance. The frequency selectivity can be further enhanced due to specific temperature increase inside the pillar on resonance. The method can potentially fill the gap of far-infrared detectors around 10$30 \mathrm{THz}$.

\section{METHODS}

In order to demonstrate the concept of plasmonic cavity absorber, microcavities are defined in moderately doped silicon by conventional microfabrication techniques combined with deep reactive ion etching (DRIE). Silicon substrates are of $270 \mu \mathrm{m}$ thickness, 2 in. diameter, and (100) crystalline orientation. They are doped with phosphorus (n-type) with a resulting dc resistivity of $0.02-0.05 \Omega \cdot \mathrm{cm}$, which corresponds to a carrier concentration of $\sim 2 \times 10^{17} \mathrm{~cm}^{-3}$ and an electron mobility of $603 \mathrm{~cm}^{2} /(\mathrm{V} \cdot \mathrm{s})$ at room temperature. The substrates are patterned with photoresist to define the annular structures covering an area of $36 \times 36 \mathrm{~mm}^{2}$ with cavity dimensions given in Figure 1. The samples are then etched using a Bosch DRIE process in a $\mathrm{SF}_{6}$ (etchant) and $\mathrm{C}_{4} \mathrm{~F}_{8}$ (passivation) fluorinebased chemistry to a depth of 60 at $2.5 \mu \mathrm{m} / \mathrm{min}$ in the regions not protected by photoresist. The photoresist is removed by rinsing in acetone and isopropyl alcohol, resulting in the plasmonic absorber samples, as shown in Figure 7.

The plasmonic absorber sample is measured with a reflection-mode terahertz time-domain spectrometer ( $\mathrm{THz}-$ TDS) at room temperature in a nitrogen atmosphere. ${ }^{28} \mathrm{~A}$ Ti:sapphire ultrafast laser is used as the pump source for the system, with a center wavelength of $800 \mathrm{~nm}$, a repetition rate of $80 \mathrm{MHz}$, and a pulse width of less than $100 \mathrm{fs}$. Both the emitter 


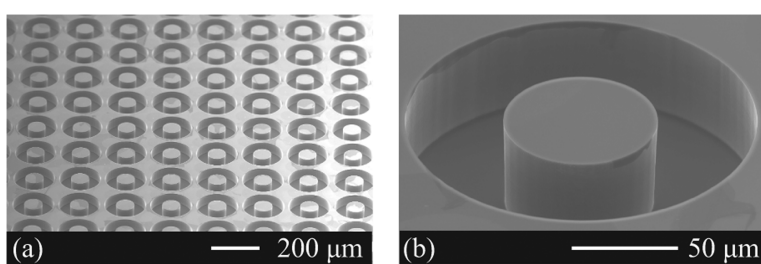

Figure 7. Scanning electron micrographs of the fabricated microcavities. (a) Partial view of the cavity arrays and (b) magnified view of a single cavity. The micrographs are taken at the tilt angle of $45^{\circ}$. The dimensions are according to the diagram in Figure 1

and detector are low-temperature-grown GaAs-based photoconductive antennas. The emitter is a Gigaoptics Tera-SED large-area interdigitated array biased with a $\pm 10 \mathrm{~V}$ square-wave operating at a chopping frequency of $10 \mathrm{kHz}$, while the detector is a Menlo Systems TERA8-1 H-dipole, with a Tydex $10 \mathrm{~mm}$ diameter hyper-hemispherical high-resistivity silicon lens. A lock-in amplifier connected to a computer is then used to acquire the modulated signal. The system bandwidth is around $2.0 \mathrm{THz}$, with a maximum dynamic range of 600 . The terahertz beam is focused down onto the sample surface at normal incidence with two off-axis parabolic mirrors, and then the reflected beam is directed into the detector via a beamsplitter and another off-axis parabolic mirror. The reflection spectrum is normalized to the reference taken from a gold mirror. To ensure the phase consistency, a motorized linear stage is used to swap between the sample and gold mirror with an accuracy of $1 \mu \mathrm{m}$ in the propagation direction.

\section{AUTHOR INFORMATION}

\section{Corresponding Authors}

*E-mail: withawat@eleceng.adelaide.edu.au.

*E-mail: sharath.sriram@gmail.com.

\section{Present Address}

${ }^{\perp}$ Department of Electronic Engineering, The Chinese University of Hong Kong, Shaitin, NT, Hong Kong

\section{Notes}

The authors declare no competing financial interest.

\section{ACKNOWLEDGMENTS}

W.W. acknowledges T. May, The Institute of Photonic Technology (IPHT), Jena, Germany, for insightful discussions on microbolometer technology. M.B. and S.S. acknowledge Australian Postdoctoral Fellowships from the Australian Research Council (ARC) through Discovery Projects DP1092717 and DP110100262. S.S. acknowledges partial support from a Victoria Fellowship. W.W. and D.A. acknowledge funding via ARC Discovery Project DP120100200. Technical assistance from $\mathrm{H}$. Ho is gratefully acknowledged.

\section{REFERENCES}

(1) Landy, N. I.; Sajuyigbe, S.; Mock, J. J.; Smith, D. R.; Padilla, W. J. Perfect metamaterial absorber. Phys. Rev. Lett. 2008, 100, 207402.

(2) Tao, H.; Landy, N. I.; Bingham, C. M.; Zhang, X.; Averitt, R. D.; Padilla, W. J. A metamaterial absorber for the terahertz regime: design, fabrication and characterization. Opt. Express 2008, 16, 7181-7188.

(3) Watts, C. M.; Liu, X.; Padilla, W. J. Metamaterial electromagnetic wave absorbers. Adv. Mater. 2012, 24, OP98-OP120.

(4) Chen, H.-T. Interference theory of metamaterial perfect absorbers. Opt. Express 2012, 20, 7165-7172.
(5) Moreau, A.; Cirací, C.; Mock, J. J.; Hill, R. T.; Wang, Q.; Wiley, B. J.; Chilkoti, A.; Smith, D. R. Controlled-reflectance surfaces with film-coupled colloidal nanoantennas. Nature 2012, 492, 86-89.

(6) Hao, J.; Wang, J.; Liu, X.; Padilla, W. J.; Zhou, L.; Qiu, M. High performance optical absorber based on a plasmonic metamaterial. Appl. Phys. Lett. 2010, 96, 251104.

(7) Liu, N.; Mesch, M.; Weiss, T.; Hentschel, M.; Giessen, H. Infrared perfect absorber and its application as plasmonic sensor. Nano Lett. 2010, 10, 2342-2348.

(8) Aydin, K.; Ferry, V. E.; Briggs, R. M.; Atwater, H. A. Broadband polarization-independent resonant light absorption using ultrathin plasmonic super absorbers. Nat. Commun. 2011, 2, 517.

(9) Tittl, A.; Mai, P.; Taubert, R.; Dregely, D.; Liu, N.; Giessen, H. Palladium-based plasmonic perfect absorber in the visible wavelength range and its application to hydrogen sensing. Nano Lett. 2011, 11, $4366-4369$.

(10) Hedayati, M. K.; Javaherirahim, M.; Mozooni, B.; Abdelaziz, R.; Tavassolizadeh, A.; Chakravadhanula, V.; Zaporojtchenko, V.; Strunkus, T.; Faupel, F.; Elbahri, M. Design of a perfect black absorber at visible frequencies using plasmonic metamaterials. Adv. Mater. 2011, 23, 5410-5414.

(11) Teperik, T. V.; Garca de Abajo, F. J.; Borisov, A. G.; Abdelsalam, M.; Bartlett, P. N.; Sugawara, Y.; Baumberg, J. J. Omnidirectional absorption in nanostructured metal surfaces. Nat. Photonics 2008, 2, 299-301.

(12) Zhang, J.; Ou, J.-Y.; MacDonald, K. F.; Zheludev, N. I. Optical response of plasmonic relief meta-surfaces. J. Opt. 2012, 14, 114002.

(13) Le Perchec, J.; Quémerais, P.; Barbara, A.; López-Ríos, T. Why metallic surfaces with grooves a few nanometers deep and wide may strongly absorb visible light. Phys. Rev. Lett. 2008, 100, 066408.

(14) Kravets, V. G.; Schedin, F.; Grigorenko, A. N. Plasmonic blackbody: Almost complete absorption of light in nanostructured metallic coatings. Phys. Rev. B: Condens. Matter 2008, 78, 205405.

(15) Popov, E.; Maystre, D.; McPhedran, R. C.; Nevière, M.; Hutley, M. C.; Derrick, G. H. Total absorption of unpolarized light by crossed gratings. Opt. Express 2008, 16, 6146-6155.

(16) Giannini, V.; Berrier, A.; Maier, S. A.; Antonio Sánchez-Gil, J.; Gómez Rivas, J. Scattering efficiency and near field enhancement of active semiconductor plasmonic antennas at terahertz frequencies. Opt. Express 2010, 18, 2797-2807.

(17) Berrier, A.; Ulbricht, R.; Bonn, M.; Gómez Rivas, J. Ultrafast active control of localized surface plasmon resonances in silicon bowtie antennas. Opt. Express 2010, 18, 23226-23235.

(18) Berrier, A.; Albella, P.; Ameen Poyli, M.; Ulbricht, R.; Bonn, M.; Aizpurua, J.; Gómez Rivas, J. Detection of deep-subwavelength dielectric layers at terahertz frequencies using semiconductor plasmonic resonators. Opt. Express 2012, 20, 5052-5060.

(19) Hanham, S. M.; Fernández-Domínguez, A. I.; Teng, J. H.; Ang, S. S.; Lim, K. P.; Yoon, S. F.; Ngo, C. Y.; Klein, N.; Pendry, J. B.; Maier, S. A. Broadband terahertz plasmonic response of touching InSb disks. Adv. Mater. 2012, 24, OP226-OP230.

(20) Withayachumnankul, W.; Shah, C. M.; Fumeaux, C.; Kaltenecker, K.; Walther, M.; Fischer, B. M.; Abbott, D.; Bhaskaran, M.; Sriram, S. Terahertz localized surface plasmon resonances in coaxial microcavities. Adv. Opt. Mater. 2013, 1, 443-448.

(21) Catrysse, P. B.; Fan, S. Understanding the dispersion of coaxial plasmonic structures through a connection with the planar metalinsulator-metal geometry. Appl. Phys. Lett. 2009, 94, 231111.

(22) Bliokh, K. Y.; Bliokh, Y. P.; Freilikher, V.; Savel'ev, S.; Nori, F. Colloquium: unusual resonators: plasmonics, metamaterials, and random media. Rev. Mod. Phys. 2008, 80, 1201-1213.

(23) Asheghi, M.; Kurabayashi, K.; Kasnavi, R.; Goodson, K. E. Thermal conduction in doped single-crystal silicon films. J. Appl. Phys. 2002, 91, 5079-5088.

(24) Maier, T.; Brueckl, H. Multispectral microbolometers for the midinfrared. Opt. Lett. 2010, 35, 3766-3768.

(25) Georgiou, G.; Tyagi, H. K.; Mulder, P.; Bauhuis, G. J.; Schermer, J. J.; Gómez Rivas, J. Photo-generated THz antennas. Sci. Rep. 2014, 4, 3584 . 
(26) Tao, H.; Kadlec, E. A.; Strikwerda, A. C.; Fan, K.; Padilla, W. J.; Averitt, R. D.; Shaner, E. A.; Zhang, X. Microwave and terahertz wave sensing with metamaterials. Opt. Express 2011, 19, 21620-21626.

(27) Maier, T.; Brückl, H. Wavelength-tunable microbolometers with metamaterial absorbers. Opt. Lett. 2009, 34, 3012-3014.

(28) Ung, B. S.-Y.; Li, J.; Lin, H.; Fischer, B. M.; Withayachumnankul, W.; Abbott, D. Dual-mode terahertz timedomain spectroscopy system. IEEE Trans. Terahertz Sci. Technol. 2013, 3, 216-220. 